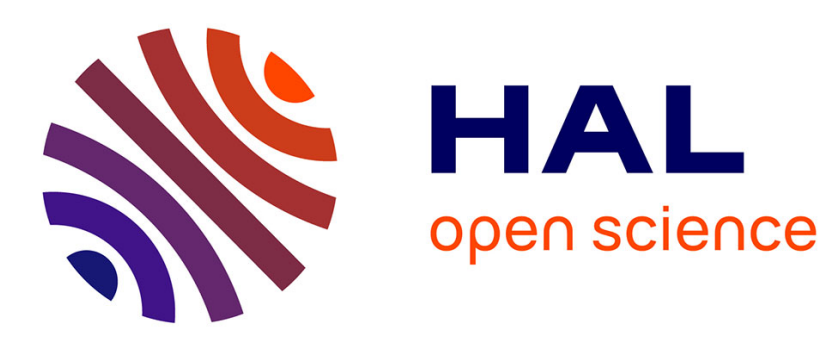

\title{
An ontological model for the reality-based 3D annotation of heritage building conservation state
}

Tommy Messaoudi, Philippe Véron, Gilles Halin, Livio de Luca

\section{To cite this version:}

Tommy Messaoudi, Philippe Véron, Gilles Halin, Livio de Luca. An ontological model for the realitybased 3D annotation of heritage building conservation state. Journal of Cultural Heritage, 2018, 29, pp.100-112. 10.1016/j.culher.2017.05.017 . hal-01982887

\section{HAL Id: hal-01982887 \\ https://hal.science/hal-01982887}

Submitted on 28 Jan 2019

HAL is a multi-disciplinary open access archive for the deposit and dissemination of scientific research documents, whether they are published or not. The documents may come from teaching and research institutions in France or abroad, or from public or private research centers.
L'archive ouverte pluridisciplinaire HAL, est destinée au dépôt et à la diffusion de documents scientifiques de niveau recherche, publiés ou non, émanant des établissements d'enseignement et de recherche français ou étrangers, des laboratoires publics ou privés. 


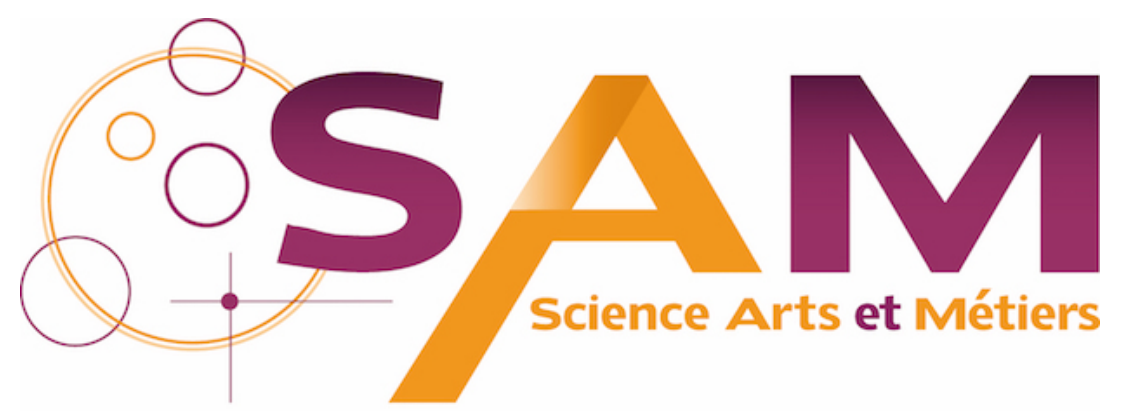

Archive Ouverte - Open Repository

\section{Science Arts \& Métiers (SAM)}

is an open access repository that collects the work of Arts et Métiers ParisTech researchers and makes it freely available over the web where possible.

This is an author-deposited version published in: https://sam.ensam.eu Handle ID: .http://hdl.handle.net/10985/14255

\section{To cite this version :}

Tommy MESSAOUDI, Philippe VÉRON, Gilles HALIN, Livio DE LUCA - An ontological model for the reality-based $3 \mathrm{D}$ annotation of heritage building conservation state - Journal of Cultural Heritage - Vol. 29, p.100-112 - 2018 


\title{
An ontological model for the reality-based 3D annotation of heritage building conservation state
}

\author{
T. Messaoudi ${ }^{1,2}$, P. Véron ${ }^{2}$, G. Halin, L. De Luca ${ }^{1}$ \\ ${ }^{1}$ UMR 3495 MAP CNRS/MCC, Campus CNRS Joseph Aiguier - Bât. Z' 31 chemin Joseph Aiguier 13009 \\ Marseille, France \\ tommy.messaoudi@map.cnrs.fr \\ livio.deluca@map.cnrs.fr \\ ${ }^{2}$ UMR CNRS 7296 LSIS, Arts et métiers ParisTech 13100 Aix-en-Provence, France \\ Philippe.veron@ensam.eu
}

Keywords: Conservation, Cultural heritage, Ontology, Data management, semantic annotation, knowledge

\begin{abstract}
The practice of restoring historical monuments requires making a conservation diagnosis that constitutes the study prior to restoration. Nowadays it is possible to describe the overall cultural heritage building with a huge amount of heterogeneous data. In the conservation domain, the data from different technics and specific protocols are produced to create new information allowing cultural heritage experts to explain the building life cycle through scientific observations. Given the great advances on technology development for the 2D and 3D digitization of heritage building, the main issue encountered by the Cultural Heritage community today in this wide domain does not concern the access to new tools for creating a rich, multi-dimensional and multiformat data collection, but the possibility to correlate these heterogeneous data in order to produce relevant information (related to consolidated knowledge) for describing the building conservation state. Another important point concerns the spatial referencing gap. Data generated by different experts through graphic (or textual) supports or other process are generally not spatialized: even if all these data refers to a common physical object (e.g. an heritage building) or to specific spatial regions of this objet (e.g. a degradation pattern on a wall) the link between these data is only based on a conceptual description of the building (without any references to their spatial reciprocal position). In the past years, many 3D digitization techniques (laser scanning, photogrammetry, etc.) have emerged allowing the domain experts to generate dense and accurate geometric representations of historic buildings. But, even if the $3 \mathrm{D}$ digitization technologies allow creating dense 3D geometric representations of an heritage artefact, the automatic processing of these raw 3D data (e.g. pointclouds, 3D mesh, etc.) does not provide any relevant information from a semantic point of view, especially when the analysis and interpretation purpose relates to the conservation analysis field. In fact, beyond geometry, the analysis and the management of a big amount of heterogeneous data within a multi-disciplinary knowledge domain, is certainly the core scientific issue in this field. In this sense, building an ontological model for the multi-disciplinary observation of the conservation state purposes seems to be a promising way for structuring semantic-aware 3D representations of heritage buildings today. This paper introduces a domain ontology model for the reality-based 3D semantic annotations of the building conservation state. By combining qualitative and quantitative descriptors
\end{abstract}


of interconnected 3D annotations (2D/3D spatial regions related to semantic concepts), this dedicated ontology integrates data, information and knowledge for describing and monitoring stone degradation phenomena in order to assist Cultural Heritage experts within the decision-making process. Our approach for the reality-based 3D annotation of heritage buildings, related to the formal representation of structured knowledge belonging to its scientific observation, represents a promising way for guiding the collaborative analysis of the conservation state towards the progressive implementation of a shared and consensual representation of this domain knowledge.

\section{INTRODUCTION AND OBJECTIVES}

In the field of cultural heritage, there is today an important challenge about building conservation state monitoring. A major question is how to structure, connect and provide access to complex data interpretation in the field of conservation? The description and understanding of the built heritage held by a large and very diverse number of documentary sources (text, graphics, voice) and analytical data (such as from the sample analysis, data from various sensors, map data from different imaging radiations, etc.). The data generation and process are conventional according the expertise domain (Architecture, mechanics, computer sciences, etc.) and all of them comprises all the historical, archaeological and constructive information required to understand the heritage structure and its evolution over time. Despite their complementary, analytic data sources are often separated from one another. So the attempt to structure, share and link these data has real meaning in the field of conservation. In addition, given the great advances on technology development for the $2 \mathrm{D}$ and $3 \mathrm{D}$ digitization of heritage building, the main issue encountered by the Cultural Heritage community today in this wide domain concern the possibility to correlate these heterogeneous data in order to produce relevant information (related to consolidated knowledge) for describing the building conservation state. Another important point concerns the spatial referencing gap. Data generated by different experts through graphic (or textual) supports or other process are generally not spatialized: even if all these data refers to a common physical object (e.g. an heritage building) or to specific spatial regions of this objet (e.g. a degradation pattern on a wall) the link between these data is only based on a conceptual description of the building (without any references to their spatial reciprocal position).

Design an ontological model for the multi-disciplinary observation of the conservation state purposes seems to be a promising way for structuring semantic-aware 3D representations of heritage buildings today. This paper introduces a domain ontology model for the reality-based 3D semantic annotations of the building conservation state. By combining qualitative and quantitative descriptors of interconnected 3D annotations this dedicated ontology integrates data, information and knowledge for describing and monitoring stone degradation phenomena in order to assist Cultural Heritage experts within the decision-making process. This article is divided into five parts. The section 2 will present several relevant principle in order to well understand manipulated notions. Then propose a state of the art about existing system that can either manage a huge amount of data thank to domain ontology or spatialize scientific observation around 3D representation. Our approach on the domain ontology design dedicated to 3D semantic annotation will be developed in section 3. The correlation engine explained in the section 4 will particularly show the merging of specific dimensions. In the section 5 will be also presented the chosen implementation strategy to build the overall information system. And finally the last section will conclude this article and will propose some related perspectives. 


\section{STATE OF THE ART : LITERATURE REVIEW}

This section will be split in two part. The first one will give some basics definitions in order to well understand the manipulated concepts exploited in the conservation monitoring issue. The second one regroups the most relevant related work proposing solutions to respond as well as possible to the given problem: works from which we will exploit benefits for the presented approach in the next section.

\subsection{Basic Definitions}

Many approaches are used today for reproducing and representing with objectivity the current state of an heritage building according to its morphological and conceptual complexity. In this section we analyze the scientific literature related to the three main domains which constitute the main dimensions of our approach: the domain ontology, the semantic annotation and then the reality-based $3 \mathrm{D}$ reconstruction.

\subsubsection{Domain ontology definition}

An ontology is used to describe, share and reuse knowledge and data between software and humans. This conceptual model is used in a lots of information system exploiting semantic web technology. But the name has been borrowed from philosophic domain and is defined as the "study of being as being" [9]. Particularly in the computer sciences domain and knowledge-based engineering, the ontology is characterized like "explicit and formal specification of a shared conceptualization". This definition shows and link a lot of specific concept: Conceptualization means that a specific expert group of the same domain want to describe and model an abstract phenomena existing in the world. "Explicit" refers to the concepts and constrains that need to be clearly defined in relation to the domain. An ontology is "formal" because it is understandable and readable by a computer allowing it to produce some reasoning between concepts thanks to defined rules. And finally it can be "shared", meaning that it gather consensual knowledge agreed by a scientific community' expert group. Thus, a domain ontology is define as a shared knowledge formalization dedicated to a specific domain.

\subsubsection{Reality-based 3D reconstruction}

The reality-based 3D reconstruction, is the technic used to create three-dimensional representation of a real object. In recent decades, there is an important demand for computer graphics triggering a great interest and change for the requirements. The creation of 3D model of heritage and archeological objects and sites in their current state requires a powerful methodology able to capture and digitally model the fine geometric and appearance details of such sites. The most common methodology followed is about image-based and laser-based approach that is nowadays well-known. Several attempts have been conducted particularly by using image manual adjustment technic, nevertheless they did not constitute a fairly accurate solution. That why several research groups have developed a technic named automated imagebased 3D reconstruction including tools for orientation and image calibration [22]. Thank to this technic it is possible to performed a huge amount of study (metrical, morphological, spatial analysis and so on).

\subsubsection{Semantic annotation}

Annotation principle is based on the linking between one entity and complementary information. The semantic annotation represents the process that associates a tag aiming to argument advanced research on a particular analysis. Furthermore, annotation is used to convert syntactical framework to knowledge structuration. Then defined annotations allow to create complex information structuration. Indeed, this structuration has a real influence on data displayable by user. Four model of semantic enrichment: by tags (keywords), by attributes (object features), by relationships (between provided resources) and then by 
ontologies ("an explicit specification of a shared conceptualization") [1].

In the domain of cultural heritage, the process of annotation on iconographic sources and more specifically on photographs helps the comprehension of a building by informing semantic information. Three main methods are currently available to annotate a 2D source: manual (annotation related with tag or term from ontology), automatic (by shape recognition), or semiautomatic (validation of a keyword proposed by the system). However these three methods only use 2D information. Regarding 3D models annotations, information can be attached to points, segments, surfaces or objects in the digital mock-up.

Recently, researchers have shown an increased interest in the use of 3D information in the image semantic annotation process.

\subsection{Related works}

Two aspects can be highlighted in the conservation domain. On the one hand several Information System propose the management of a large amount of data type so the qualitative aspect is the most exposed. One the other hand few systems are based on the quantitative aspect. And then in general, there is little studies relating to these two main aspects within an integrated approach.

\subsubsection{Documenting Cultural Heritage objects by a domain ontology}

The research group SeCo (Semantic Computing) has designed an ontology named MAO (Museolan Ontologia). The latter has been created for the content description such as tangible and intangible object. MAO structure data used by an application located into "the semantic portal for Finnish Culture Kulttuurisampo". One of the first public release, contains data from twenty-odd sources such as museums, library, archives, and so on. This application integrates contents produced by a large domain panel like painting, sculpture, art of drawing fields, and from web page, data about place, historical events, cultural sites, etc. Those data are related to each other thank to a domain ontology [11]. The intrinsic information and images metadata are directly linked with location data displayed on a cartography web site such as google map. It is possible only by the use of an ontology because this data is complex to manage just with a relational database. To conclude on this first example, the research group have design an ontology that allow to users to visualize a lots of heterogeneous data linked between them and then give some knowledge about the observable entities. However, the ontology and the overall system don't manage the 3D information.

\subsubsection{MONDIS : Conservation Domain}

MONDIS which stand for MONument Damage Information System, is dedicated to heritage building conservation, also manage alteration phenomena. Indeed the domain ontology is based on an automatic coordination intersecting: cultural heritage alteration documentation, its diagnosis, and the intervention for possible restorations [5].

A model realized on OpenRDF software (an ontology editor) present several features divided by five distinct components, that each have their own characteristics, related to the current main element: Cultural Heritage Object. Firstly, the direct relationships focus on the component about "building description and components" defined like being architectural elements definition giving information about functional and physical characteristics.

Secondly, the element influencing the object condition: events related to the cultural heritage object. The latter takes in consideration the natural disaster (risk activation and hazard, like flooding or eartherquake), because such conditions can considerably change morphologically the object. Thirdly, the "Alteration diagnosis and intervention" component is represented by the reciprocal relationships presents between alteration phenomena, degradation agent (defined like phenomenon carrier like water or salts) and their manifestations. Then the restoration intervention will be applied in relation to either by acting on a specific alteration 
or by deleting the concerned agent. The "risk assessment" concerns the prevention aspect: which part of the study building is considered like risk in relation to a given alteration. So with the "measures assessments" it is possible to quantify and qualify several measurable aspect allowing to create a dedicated documentation (height, thickness, alteration's surface extension).

MONDIS has been designed to ease the reasoning computing between different document sources concerning the heritage building degradations: and therefore cross and create new information and new knowledge.

To conclude, this complete work is very interesting because this ontology manage a lots of heterogeneous data about the building conservation state. In addition it is most focus on a qualitative aspect. However MONDIS doesn't use spatialized annotations giving accuracy metric information. In addition, it doesn't manage information about material chemical analysis. This is the reason why we would like enhance it, using it as a starting point of our work.

\subsubsection{D databases dedicated to conservation}

In the framework of cultural heritage conservation project, information systems exist to describe reality-based 3D artifacts. Two relevant example that show the importance and the usefulness to spatialize conservation information.

The first one is an interactive three dimensional database applied to the conservation of a painted chapel. This system allows to the user to spatialize some observation directly in a 3D scaled entity [26].

In a SACRE Project, a tool dedicated to the digital documentation and the monitoring of stone alteration has been designed [25]. Thanks to this system, we can easily make some annotations on orthoimages linked with the 3D mesh and relate them with a concept from a taxonomy. Colored regions are realized directly on 2D textures and associated with a term available in a relational database. The main goal is to provide to the conservation experts a solution for observing distribution of different description layer. Carried out Annotations are compared between them by the queries: like statistical analysis, spatial distribution of cartography on the fly, and intersection between them. The 3D region spatial referencing is possible but managed data is not structured by an ontology. This work is a first and interesting attempt to annotate but it is not enough to describe and represent the overall scientific process for the cultural heritage building conservation monitoring.

\subsubsection{Related works statements}

The analysis of these related works allows to emphasis interesting remaining scientific issue. The first one (the semantic portal for Finnish Culture Kulttuurisampo) uses a domain ontology but the user cannot annotate cultural object and spatialize scientific observation as well. The second one (MONDIS) exploits domain ontology particularly dedicated to building degradation monitoring but it is not also possible to spatialize semantic annotation and extract quantitative morphological information. And finally the two spatial referencing 3D databases dedicated to conservation can manage spatialized regions around 3D representations but terms are not correlated and managed by a domain ontology. 


\section{MAIN APPROACH : A DOMAIN ONTOLOGY DEDICATED TO 3D SEMANTIC ANNOTATION}

The proposed approach will deal with two emphasized aspect involved in the cultural heritage conservation analysis: The quantitative (exploited through acquisition and data analysis) and qualitative aspect (based on expert knowledge interpretation by using description terms), that are complementary, together constitute a relevant solution to describe as effectively as possible the heritage building conservation state. The main issue in this domain is that the gap of solutions for establishing a functional relationship between them. Then the central question to be explored in this field becomes: How to design and implement a formal representation of a conservation domain knowledge able to correlate quantitative and qualitative descriptions of complex building? And how to ontologically relate such diverse aspects within a spatiotemporal information system?

This approach (figure 1) is mainly based on the integration of three characteristic dimensions. Firstly of all a semantic dimension presented in section 3.1, explaining the domain ontology design organizing concepts used by expert for describing the conservation state. Information generation will be extracted through the crossing of these concepts related by specific relationships. Secondly, the spatial dimension explained in section 3.2 describes the using of a Reality-based 3D annotation system particularly by exploiting in situ acquisition, allow to spatialize annotations performed by expert into a 3D geometric representation managing different temporal state. Finally, the morphological dimension that will be developed in section 3.3, will explain geometrical information extraction attached to a 3D representation linked to 2D annotations allowing to connect ontology concepts (e.g. "pillar") with morphological descriptors, useful to analyze and classify. 


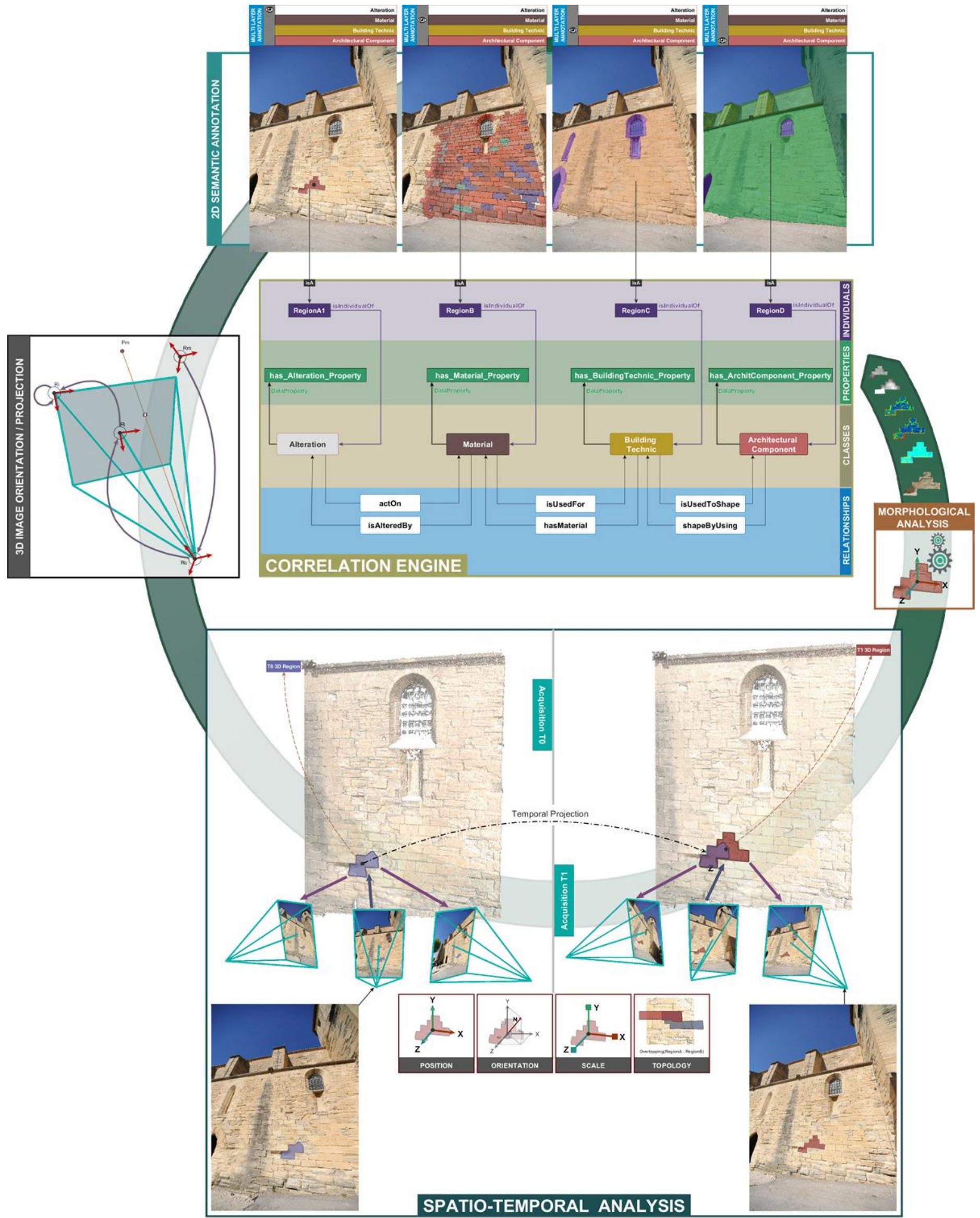

Figure 1. Multi-dimensional semantic correlation engine 


\subsection{The Semantic dimension}

The semantic dimension is based on the overlapping of four thematic description layers given by our ontology core: material, building technic, architectural component, and degradation phenomena. This important action relating annotation to a concept (by an Uniform Resource Identifier) constitutes the main link between a semantic annotation environment (see section 3.2) and the domain ontology.

\subsubsection{The domain Ontology design}

A domain ontology asks a particular attention and important thoroughness in its conception. The progressive construction of our domain ontology design is based on the Lassila's method [13]: the ontology design process required several domain experts (architects, conservation scientists and material engineers) involved in our project [7] to consensually validate a controlled vocabulary [10] which terms, as well as their meaning, will remain stable during the whole process and by using existing glossaries [13] [18]. Two glossary types are exploited in our project: Illustrated glossary on stone deterioration patterns [3] and architecture vocabulary [20]. This is the way how we have modeled our thematic thesaurus especially for the building technic, architectural component, material and alteration. Such documents constitute references for the domain scientific community. Following this step a first thesaurus (for each description layer) is built, in order to precisely define the complex and structured relationships between the terms and then obtain a taxonomy. At this step a lightweight ontology can be created up to obtain progressively a heavyweight ontology by adding more terms, rules, constrains, and axioms.

As example, the red annotation (shown in the figure 1), constitutes (is-a) an instance/individual of the "spalling" alteration class: a specific stone alteration. As expressed in section 3.2, the semantic annotation process instantiates "individuals" of the ontology classes by spatializing scientific observation carried on $2 \mathrm{D} / 3 \mathrm{D}$ representations. In the next figure (see figure 2) is presented the object properties (relationships) present between them. The instance typed into "spalling" class is linked with an argumentation, which justify the observation, but also with quantitative data extracted from the morphological and spatial dimension (respectively in section 3.2, and in section 3.3). According to the domain expert knowledge, the consensual cultural heritage conservation description can be identified through these four necessary and sufficient classes related between them by specific object properties. In the masonry building domain "a material is used for a specific building technic and itself is used to shape a relevant architectural component. And then alteration act on material" (figure 2) [17]. This model proposes inverse object properties allowing to ensure a certain flexibility in the information retrieval point of view. In this way it is possible to cross every available data linked with each annotation type belonging to the corresponding concept by involving the whole of ontology core.

\subsubsection{CIDOC-CRM integration}

The CIDOC-CRM which stand for International Council of DOCumentation - Conceptual Reference Model provides definitions and formal structure for describing the implicit and explicit concepts and relationships used in Cultural Heritage Documentation. This conceptual model, standardized ISO, has been initiated after a long effort to create a unified, harmonized relational database model shown impossible. In order to align our work to a wider research community effort, we mapped some classes of our ontology with this model [6].

CIDOC-CRM proposes extensions covering characteristic domains. For our overall ontology, we exploited and merged three of these: the CIDOC-CRM core (which provides the common and extensible semantic framework), CRMsci for the Scientific Observation, CRMdig for the data provenance and CRMinf for the Argumentation process. 
Figure 2 shows the mapping between our ontology and CIDOC_CRM extensions [27].

As example, in figure 2, the Scientific Observation (S4_Observation) is the carrier of every information about the selected annotation(s). In the proposed approach, the semantic annotation process represents a "scientific observation" carried on the studied building. Scientific observations are then linked with the set of concepts related to stone degradation phenomena (with explicit links to the ICOMOS Glossary).

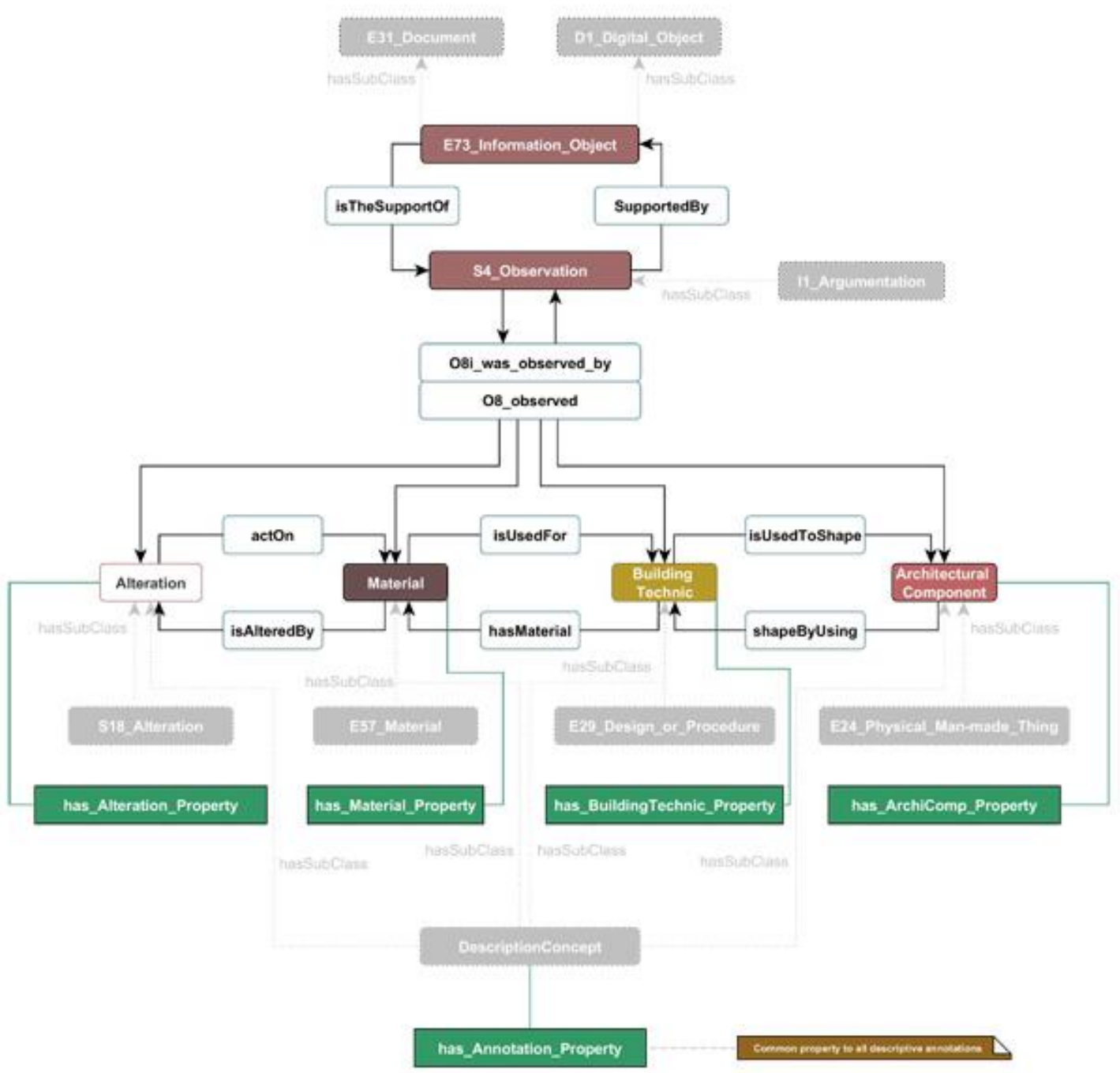

Figure 2. Ontology core mapped within the CIDOC-CRM Model

\subsection{The spatial dimension}

Our reality-based 3D annotation approach [15] allows spatializing 2D/3D regions around a consistent 3D point-based representation (a point cloud generated by multi-view photogrammetry) [22].

The proposed approach for performing reality-based 3D annotation is based essentially on a set of tools and procedures concerning the on-going development of an automated imagebased 3D reconstruction method [4]. The process consists of an automated calibration and orientation of images, a dense multi-view correlation which leads to several image generation in order to compare the morphological deterioration patterns (figure 3 ). 


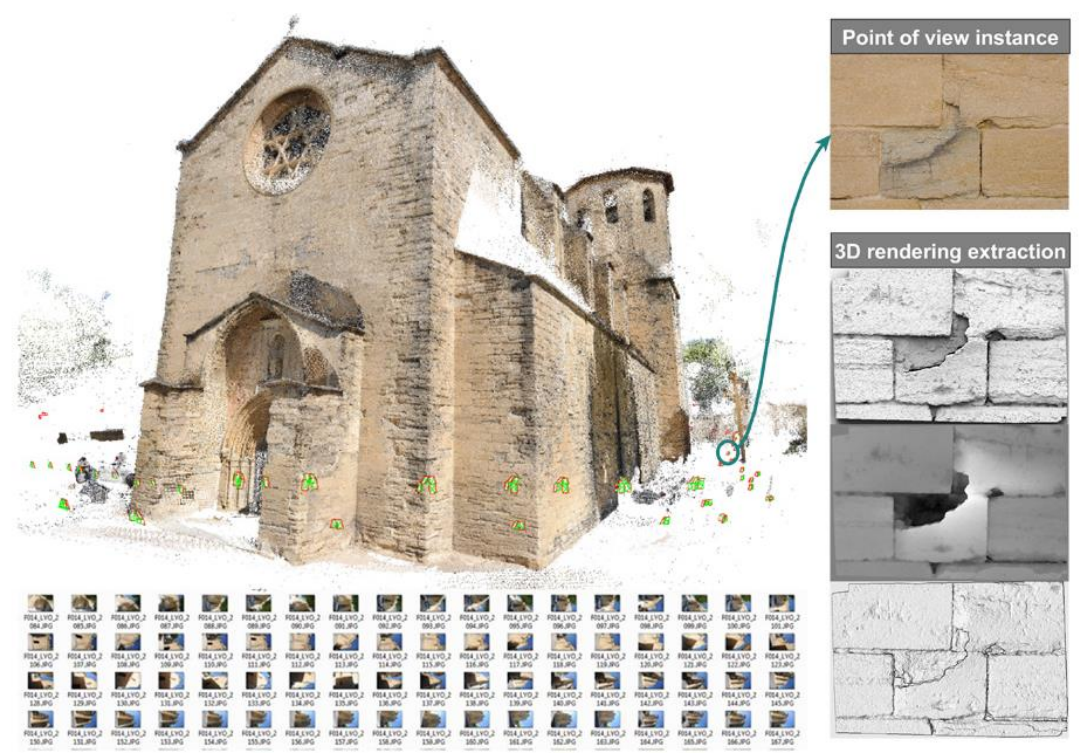

Figure 3. Image-based modeling with possible image generation

By this method, each $2 \mathrm{D}$ annotation on a spatialized image is linked to a corresponding $3 \mathrm{D}$ region used for extracting morphological attributes (see section 3.3). In fact, as $2 \mathrm{D} / 3 \mathrm{D}$ images contain a high level of information about geometric shape and visual appearance, within an annotation context become a flexible and effective support for aggregating the concepts of the semantic dimension (section 3.1). A 2D/3D bijective linkage is used for propagating semantic annotations trough the set of images, so that a simple $2 \mathrm{D}$ annotation on one image can be reprojected on other annotations (related to the same spatial area) added on other images for consequently extracting overlap degree values.

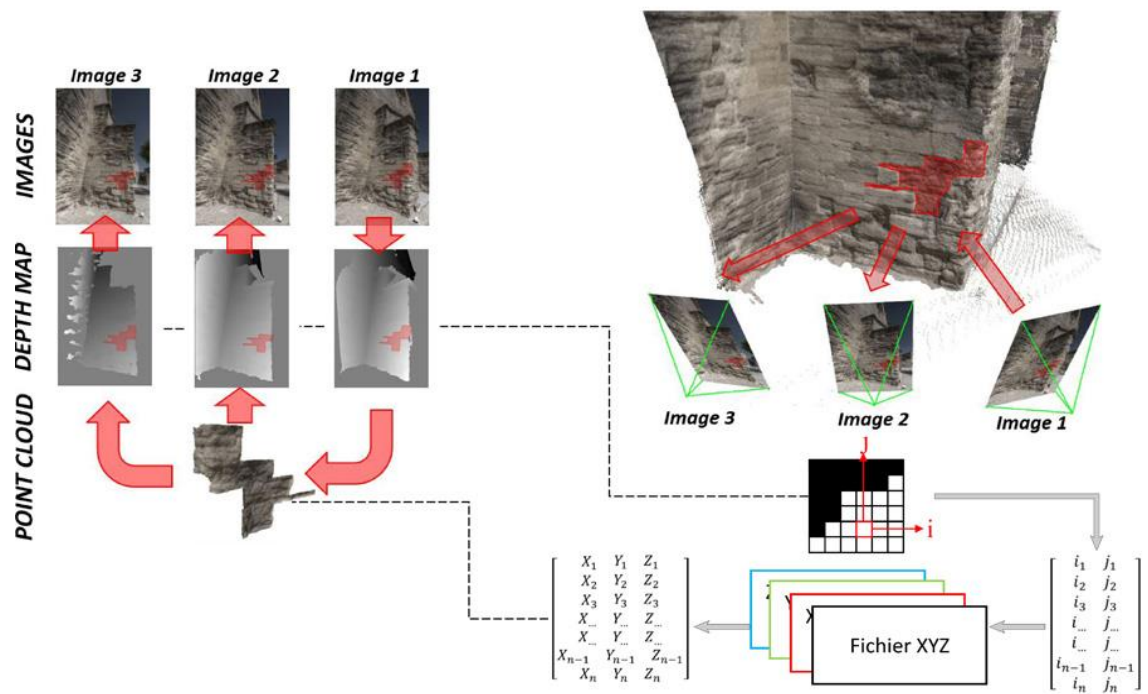

Figure 4. Transfer of annotation of a set using 2D/3D relation [15]

The process is based on two steps (see figure 4). First, when an image of the set is annotated, a 2D-to-3D projection extracts the corresponding 3D spatial region (by computing a point cloud) of the drawn area. Second, the 2D corresponding areas on other images of the set are then retrieved by a 3D-to-2D projection.

This process can be easily transposed on multiple images for defining the area to annotate 
before transferring the annotation on all images. In addition, it is possible to add new photograph acquisitions with different conservation state. As a consequence, this method provides a simple and fast way to annotate 2D images as well as a geometric method for spatializing these annotations in 3D. The annotation process does not apply only on one set of images but also on different time of acquisition of the same real object. This method allows particularly to add some other data set showing building at diverse temporal states, adjusted in the same spatial reference. So the performed annotation can be re-projected on other temporal image sets and then monitor, compare, analyze the evolution of deterioration patterns [21]. As explained in the section 3.3, the 2D/3D regions are also a relevant support for carrying out spatial and morphological analysis of the collected annotations.

\subsection{Morphological dimension}

As explained in the previous section, our annotation process produces a 3D point-based representation of each $2 \mathrm{D}$ region. This $2 \mathrm{D} / 3 \mathrm{D}$ segmentation approach can be combined with point cloud analysis approaches for extracting relevant information from the identified regions by morphological analysis. A recent work about these aspects shows that a semanticallystructured 3D point cloud can be used as support for querying quantitative data for conservation purposes [19]. By this method, each 2D annotation on an oriented image is used for extracting 3D geometric descriptors which becomes quantitative attributes for the selected ontology concept. 3D point-cloud analysis allows to extract basic spatial information (such as position, color, and orientation) of each region as well as some morphological descriptors by shape analysis approaches (such as occlusion, curvature, roughness, etc.). Our ontological model provides links for recovering these descriptors as well for linking them to the annotation concepts by a correlation engine (see next section). As an example, Figure 5 shows an image used for the manual annotation of the degradation of a wall (on the left) and the correspondent 3D point cloud used for computing a "material loss" descriptor (based on a Ransac analysis [19]). Then, each 2D annotation linked to the "degradation layer" of the ontology recovers a "material loss" value coming from the 3D point cloud analysis.

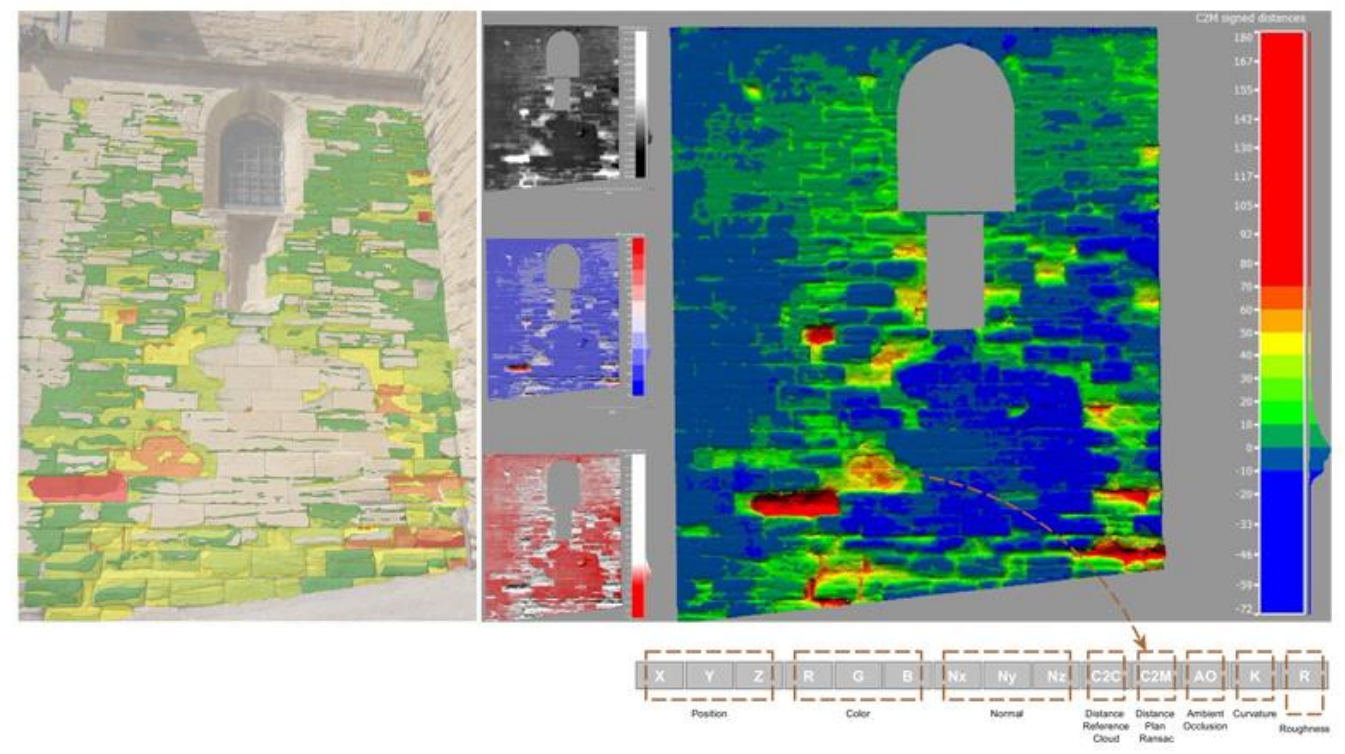

Figure 5. Traditional annotation at the left and automatic geometric analysis at the right (Ransac analysis) [4] [19] 


\section{MULTI-DIMENSIONAL CORRELATION OF SEMANTIC ANNOTATIONS}

\subsection{The ontology-based documentation process}

As highlighted by the presentation of the general architecture of our approach (section 3), the proposed domain ontology model is strongly interconnected to the annotation approach by introducing a general framework for monitoring scientific observations carried out on the heritage building analysis. Our ontology-based documentation process drives the on-going implementation of an information system built on a web architecture (see section 5). The process is composed by four main steps represented respectively by four activities that can be realized by the same or different actors (see figure 6) within a collaborative framework.

At T1 (Time 1), an actor adds a direct acquisition (such as a set of photographs) into the system. Then he provides an annotation support for performing observations and analysis.

At T2, an actor (the same or another one), adds some annotations on this analysis support by drawing $2 \mathrm{D}$ regions.

At T3, process extracts spatial relationships (by 2Dto3Dto2D projection) as well as morphological features inherent to the annotated region.

At T4, an actor assigns a concept to the drawn annotation by selecting it among the four top classes of the domain ontology: "Material", "Architectural component", "Building Technic", and then "Alteration".

At T5, an actor provides the argumentation (scientific justification) related to the annotation process, also by indicating the related references and/or resources (documentary sources, onsite observation, analytical data, etc.).
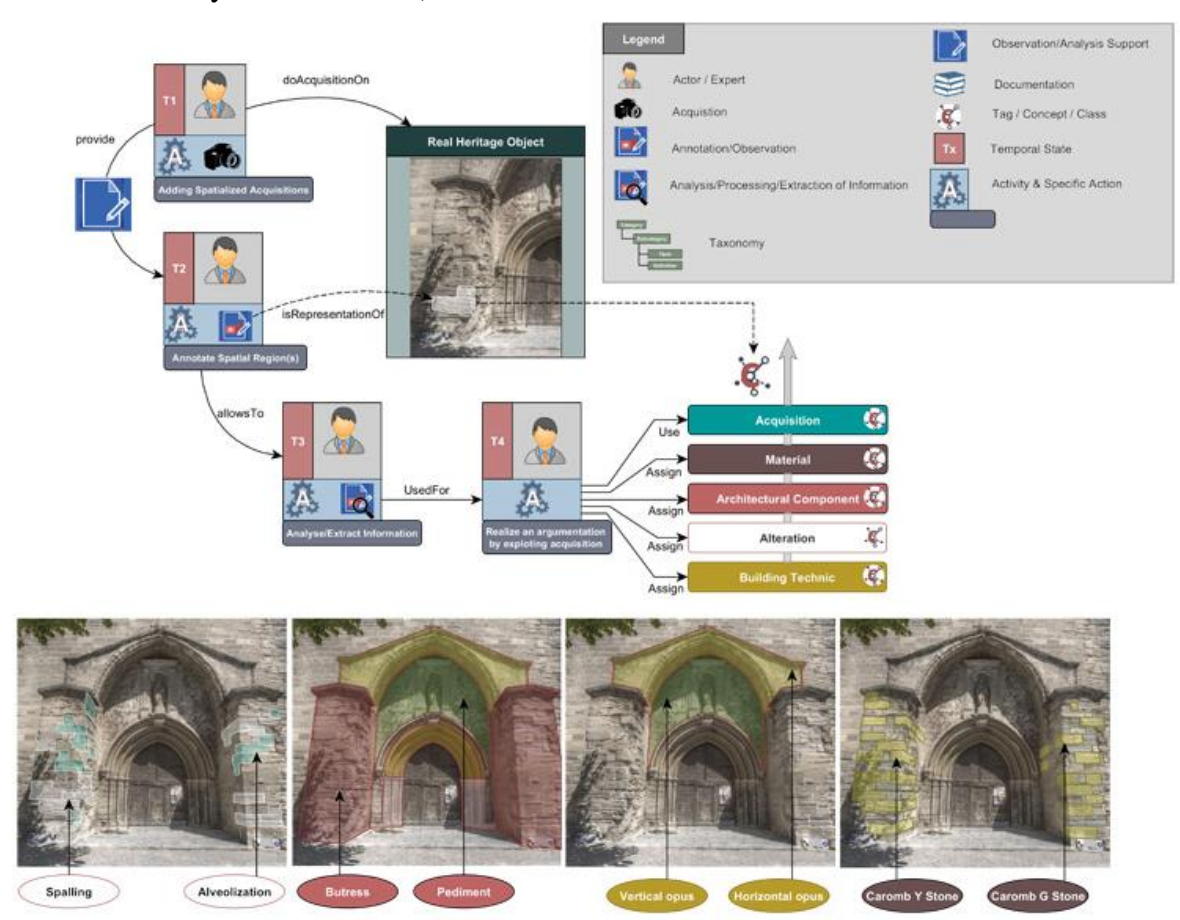

Figure 6. General approach with examples

\subsection{The correlation engine}

The system is mainly be based on reality-based 2D/3D annotation produced by an automated image-based modeling. We don't want just only retrieve raw data but also document how they have been acquired. Consequently we can document this type of acquisition (e.g. image) by using the CRMdig extension (Figure 7). For instance, acquired images on which the annotation will be done is transformed through a 3D image Orientation process associating it 
specific features. Our ontology provides the opportunity to document it for precisely monitoring the data provenance.

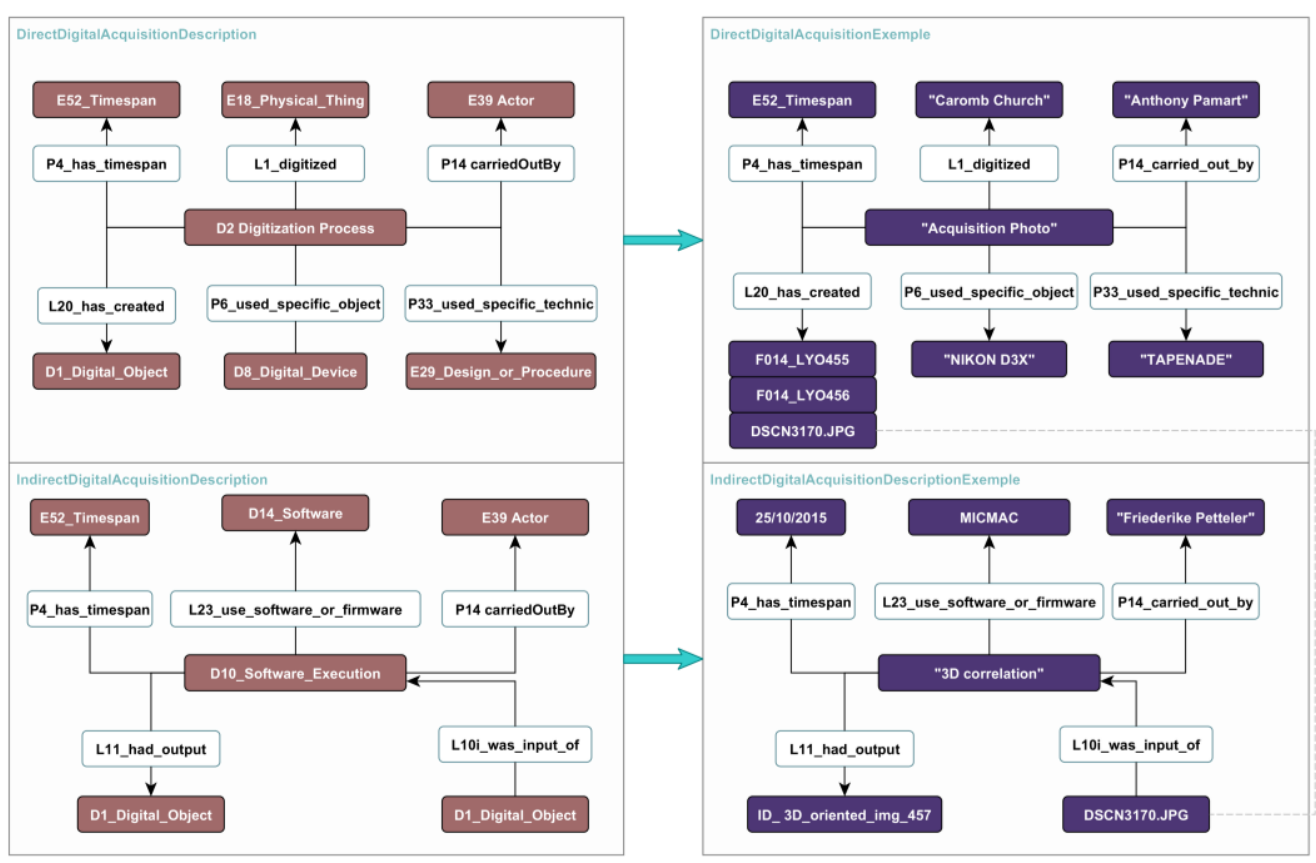

Figure 7. Acquisition description: concept to the left, example to the right

The spatialized 3D image is associated with a type available in our ontology "3D oriented images". Through the spatial dimension and the 3D image orientation process, 3D descriptors can be extracted. Therefore the annotation process starts : regions can be drawn on the images (T2) and propagated by 2D/3D projection (T3), linked to concepts of the ontology according to the above mentioned description layers (T4) and accompanied by an argumentation (T5). As explained in section 3, this annotation process (T2 to T5) can be used for carrying out semantic descriptions of several aspects of the building conservation state (also in a collaborative way) within a consistent geometric reference system. This aspect represents the main potential of our approach: by combining the 2D overlapping of the image regions with their 3D spatial referencing and morphological analysis, the annotation process allows establishing multi-dimensional correlations among the domain ontology concepts.

As an example (see figure 8), an actor draws a red 2D region on an image and assigns a concept belonging to the degradation class of the ontology (e.g. "spalling"). This region becomes an individual of the sub-class (spalling) and its spatial and morphological properties are extracted.

Then, on the same spatialized images set, another actor (or the same one) draws another region on the same area and assigns a concept belonging to the "architectural components" class of the ontology (e.g. "pillar"). And so on.

As explained in section 3.2 , the $2 \mathrm{D} / 3 \mathrm{D}$ annotation process allows spatializing all these annotations within a unique geometric reference system by providing an essential framework for exploring semantic correlations. In fact, the spatial overlapping of the 2D/3D projected annotations allow to establish semantic links within the domain ontology.

As an example, in figure 8 the spatial overlapping of the annotations belonging to the 4 description layers produces the following linkages: RegionA1 (class Alteration : subclass Spalling) actsOn RegionC (class Material : subclass Limestone) that isUsedFor RegionB (class Building Technic : subclass RegularSimpleOpus) that isUsedToShape RegionD (class Architectural Component : subclass Wall)". The correlation engine provides a way for 
crossing these regions and extracting the related information qualitative and quantitative information (figure 8). In that way expert will be able to do scientific observation on several region at the same time and then propose an example about the degradation cause at a given timespan. Previous observations involving the spalling concept will be related with the current one to realize statistical analysis and then through SPARQL Query extract information on the alteration shape. This aspect represents an important perspective for studying occurrences (e.g. the relation between degradation phenomena, materials, etc.) as well as for characterizing (by spatial and morphological attributes) the domain ontology concepts. In this sense, our ontology-driven annotation framework provides a great opportunity for the progressive building of a collection of semantically and morphologically characterized individuals (e.g. several annotations of the "spalling" phenomenon annotated in several buildings) belonging to a shared concept "class degradation: subclass spalling" on the domain ontology.

Some data have to be input manually for instance the carrier name (e.g. material property often found in the documentary sources) where the stone has been extracted [12]. Indeed the expert can assign a term to his drawn annotation and argument it. In fact, some other experts can approve observation (argumentation and assignation) and give more argumentation for a specific annotation by adding a new observation. However it is also possible to disapprove the observation and propose another suggestion.

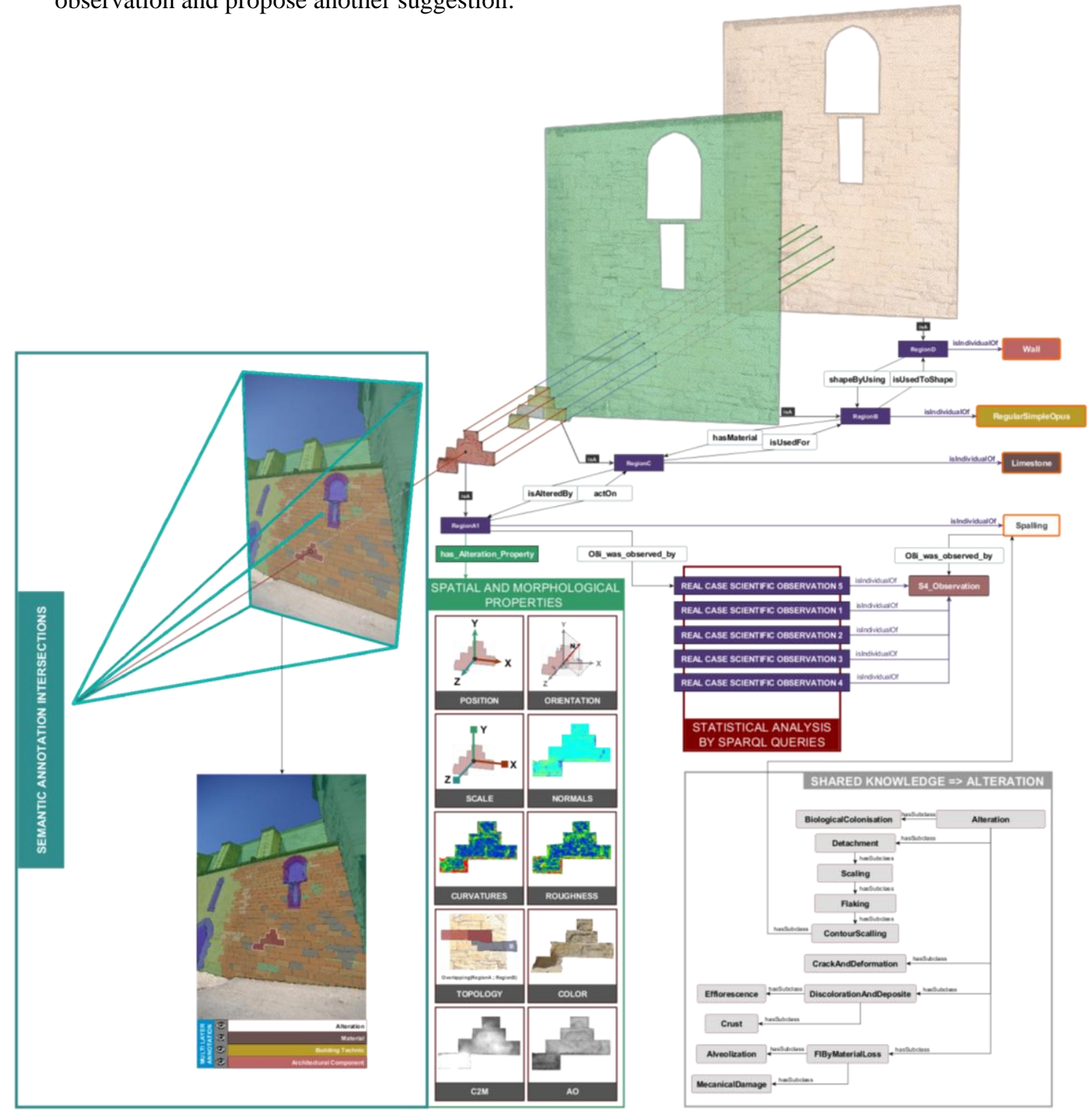

Figure 8. Semantic annotation intersection 
This model (figure 8) is the subject of an IT development which the plan is presented in section 5. The innovation of this approach is that this is the first time we bring together these three dimensions within the same information system.

\section{INFORMATIC IMPLEMENTATION PLAN}

This section present development environment and the different platform's components (figure 9). First of all the correlation engine presented in the previous section is based on a web tool which is already in implementation. The latter give the possibility to insert temporal image sets (T1), allows to carry out annotations on images (T2) and then propagate them by 2Dto3Dto2D projection (T3). These basic functions are already implemented which generated data are stored into a dedicated database. The appellation step (T4) will be managed by establishing of a plugin named jOWL, proposes to choose terms among a controlled vocabulary (integrated into the overall ontology) accompanied of their URIs [24]. The annotation will be associated with a concept and will be integrated in the semantic correlation managed by the ontology designed with Protégé Tool (OWL API). The correlation engine will be supported by the ONTOP plugin that will link existing database with the domain ontology through SQL/SPARQL mappings [2]. Then SPARQL query from ONTOP will allow us to extract relevant information through statistical analysis [23]. For visualizing available individuals and better understand their complex relationships, we will exploit the VOWL plugin based on the user-oriented visualization of ontologies [14]. The whole of conceptual model could be enriched by adding new concepts, or new controlled vocabularies (so for integrating new share knowledge), we will use functions from web protégé creating a collaborative platform to add/update/delete entities. Finally, information extracted from the semantic correlation engine will be store in a triplestore named GraphDB supplying an endpoint to do SPARQL Query.

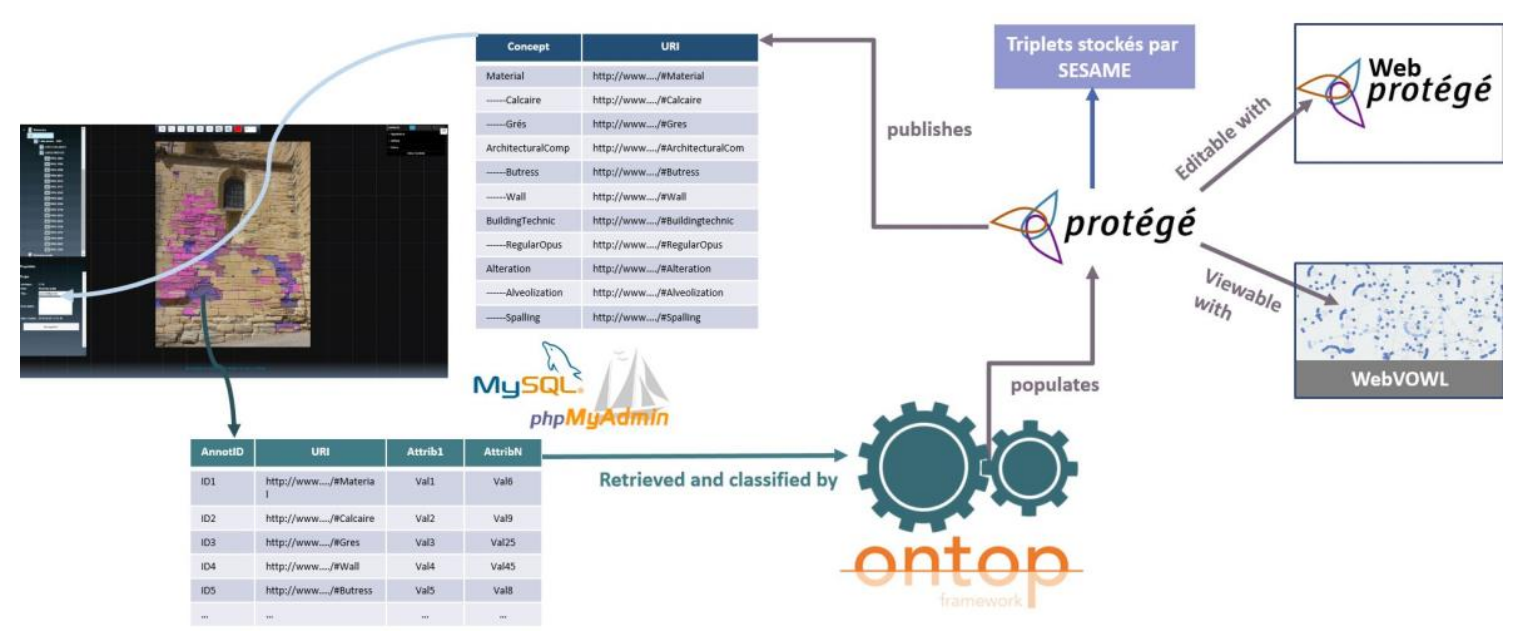

Figure 9. Implementation plan schema

\section{CONCLUSION, PERSPECTIVES}

This paper introduced a domain ontology model for the reality-based 3D semantic annotations of building conservation states. By merging the three specific dimensions (semantic, spatial, morphological) we take in consideration the qualitative and quantitative aspects. The proposed semantic correlation engine allows conservation experts in a collaborative framework to obtain information about spatially referenced regions around a reality-based $3 \mathrm{D}$ representation 
on the one hand, and, on the other hand semantically classify them into a knowledge-based system. Through a geometrical analysis process and thanks to the appellation step, the user can instantiate individuals with its own features. In addition, the spatial overlapping of the annotations belonging to the four thematic layers produces a linkage allowing to extract (through SPARQL query) data and information relating to each description class. These aspects represent an important perspective for the study of occurrences (e.g. the relation between degradation phenomena, materials, etc.) as well as for characterizing (by spatial and morphological attributes) the domain ontology concepts. This system consents the information correlation of annotations of a common physical object as well as its correlation with annotations from other heritage buildings. Another aim will be also to monitor quantitatively the evolution of deterioration patterns in different resolutions, starting from images that were taken from various points of view, with different expositions and in dissimilar time periods. By combining qualitative and quantitative descriptors of interconnected 3D annotations this dedicated ontology integrates data, information and knowledge for describing and monitoring stone degradation phenomena in order to assist Cultural Heritage experts within the decisionmaking process. The future challenge will be also to perform on situ the data acquisition and the correlation engine thanks to a mobile device in order to provide direct results for the expert [16]. This ontology still needs to be completed but the innovative integration of the main components already allows us to introduce the notion of "informative continuum" as a key for interconnecting spatialized and semantically enriched photographs to populate a knowledge base on the Cultural Heritage building degradation.

\section{ACKNOWLEDGEMENTS}

We would like to thank the partners involved into the MONUMENTUM Project that permits me to publish this work: particularly the CICRP (Centre Interdisciplinaire de Conservation et de Restauration du Patrimoine), PRISME (Centre de Recherche en Matière Divisée), LMGC (Laboratoire de Mécanique et Génie Civil) to give us data and opportunity to model the domain ontology. We also would like to thank Amelie Benard, Romina Nespeca and Philippe Bromblet responsible for the Caromb's church case study.

\section{REFERENCES}

[1] P. Andrews, I. Zaihrayeu, J. Pane: A Classification of Semantic Annotation Systems, Semantic Web, IOS Press Amsterdam, Vol.3, Issue 3, pp 223-248, August 2012.

[2] I. Astrova, A. Kalja: Automatic transformation of SQL relational databases to OWL ontologies. In: WEBIST, vol. 2, pp. 131-136 (2008).

[3] P. Bromblet, J. M. Vallet, and V. Verges-Belmin: Illustrated glossary on stone deterioration patterns. Monuments and sites, 2008.

[4] A. Benard, Report Internship: Caromb church study, Documentary research and cartography analysis, July 2015.

[5] R. Cacciotti, M.Blaško, \& J.Valach (2015). A diagnostic ontological model for damages to historical constructions. Journal of Cultural Heritage, 16(1), 40-48.

[6] M. Doerr: "The CIDOC CRM, an Ontological Approach to Schema Heterogeneity". In: Proceedings of Semantic Interoperability and Integration, 2005.

[7] L. De Luca, M. Bagneris, C. Chevrier, T. Ciblac, J.-M. Vallet, P. Bromblet, M. Pierrot-Desseilligny, X. Brunetaud, M. Al Mukhtar, F. Dubois, F. Cherblanc and R. Jourdan (2014): MONUMENTUM: Digital modelling and data management for the conservation of masonry structures. ICOMOS General Assembly, Florence, 10-15/11/2014.

[8] N. Guarino, \& C. Welty: Evaluating ontological decisions with OntoClean. Communications of the ACM, 45(2), 61-65, 2002.

[9] T. R Gruber: "Toward Principles for the Design of Ontologies Used for Knowledge Sharing". International Journal Human-Computer Studies 43, p.907-928, 23 August (1993).

[10] N. Hernandez: "Ontologie de domaine pour la modélisation du contexte en recherche d'information".

[11] E. Hyvönen: "Publishing and Using Cultural Heritage Linked Data on the Semantic Web", Synthesis Lectures on the Semantic Web: Theory and Technology, Morgan \& Claypool, pp.1-119, (2012).

[12] S. Janvier-Badosa, K. Beck, X. Brunetaud, A. Guirimand-Dufour \& M. Al-Mukhtar: Gypsum and spalling decay mechanism of tuffeau limestone. Environmental Earth Sciences, August 2015, Volume 74, Issue 3, pp $2209-2221$. 
[13] O. Lassila, R. R. Swick: "Resource description framework (rdf) model and syntax specification w3c recommendation", (1999). Recommendation 22. February 1999. http://www.w3.org/TR/1999/REC-rdf-syntax-19990222/, 1999.

[14] S. Lohmann, S. Negru, F. Haag, T. Ertl: VOWL 2: User-Oriented Visualization of Ontologies, Knowledge Engineering and Knowledge Management, Volume 8876 of the series Lecture Notes in Computer Science pp 266-281

[15] A. Manuel, L. De Luca, and P. Véron, (2014). A Hybrid Approach for the Semantic Annotation of Spatially Oriented Images. International Journal of Heritage in the Digital Era, 3(2), 305-320.

[16] T. Messaoudi, A. Manuel, E. Gattet, L. De Luca, P. Véron: "Laying the foundations for an information system dedicated to heritage building degradation monitoring based on the 2D/3D semantic annotation of photographs" EUROGRAPHICS Workshops on Graphics and Cultural Heritage (2014).

[17] T. Messaoudi, L. De Luca, \& P. Véron (2015, September). Towards an ontology for annotating degradation phenomena. In 2015 Digital Heritage (Vol. 2, pp. 379-382). IEEE.

[18] R. Navigli, P. Velardi: "From Glossaries to Ontologies: Extracting Semantic Structure from Textual Definitions", Series information for Frontiers in Artificial Intelligence and Applications, IOS Press, pp. 71-87, (2008).

[19] R. Nespeca, L. De Luca: Analysis, Thematic Maps and Data Mining From Point Cloud to Ontology for Software Development, ISPRS, (2016).

[20] J. M. Pérouse de Montclos: "Architecture : méthode et vocabulaire”, Monum, éditions du patrimoine, 2004.

[21] F. Peteler, E. Gattet, P. Bromblet, O. Guillon, J.M. Vallet, L. De Luca: Analyzing the Evolution of Deterioration Pattern : A First Step of an Image-Based Approach for Comparing Multitemporal Data Sets, Digital Heritage International Congress (DigitalHeritage), Sep 29-Oct 2, Granada, Spain, 2015.

[22] M. Pierrot-Deseilligny, L. De Luca, and F. Remondino (2011). Automated image-based procedures for accurate artifacts 3D modeling and orthoimage generation. Geoinformatics FCE CTU, 6, 291-299.

[23] M. Rodriguez-Muro, R. Kontchakov, \& M. Zakharyaschev: Ontology-based data access: Ontop of databases. In The Semantic Web-ISWC 2013 (pp. 558-573). Springer Berlin Heidelberg.

[24] V. Srivastava: Methods to visualize ontology: To obtain the degree Bachelor of Science in Information Management Koblenz, 14th July 2011.

[25] C. Stefani, X.Brunetaud, S. Janvier-Badosa, K. Beck, L. De Luca, and M. Al-Mukhtar (2014): Developing a toolkit for mapping and displaying stone alteration on a web-based documentation platform. Journal of Cultural Heritage, 15(1), $1-9$.

[26] J. M. Vallet, L. De Luca, M. Feillou, O. Guillon, and M. Pierrot-Deseilligny (2012). An interactive 3-dimensional database applied to the conservation of a painted chapel. International Journal of Heritage in the Digital Era, 1(2), 233-250.

[27] E. Vassilakaki, D. Kyriaki-Manessi, S. Zervos, \& G. Giannakopoulos (2014, September). CIDOC-CRM extensions for conservation processes: a methodological approach. In 4th international Conference on Integrated Information, Madrid, Spain. 